\title{
GENERATING FUNCTIONS FOR RELATIVES OF CLASSICAL POLYNOMIALS
}

\author{
P. D. BARRY AND D. J. HURLEY
}

(Communicated by Richard R. Goldberg)

\begin{abstract}
For several classical polynomials $u_{n}(x)$ satisfying a second order linear differential equation $D_{n}(x)$, there is a generating function $u(x, t)=$ $\sum_{n=0}^{\infty} u_{n}(x) t^{n}$. We provide expansions $v(x, t)=\sum_{n=0}^{\infty} v_{n}(x) t^{n}$ where $v_{n}(x)$ is a second solution of $D_{n}(x)$.
\end{abstract}

1. Introduction. For the derivation of generating functions we refer to [4 and 3]; for lists of them we refer to [2], and for orthogonal polynomials in particular to [1] also. There are very well-known generating functions $u(x, t)$ for the Hermite polynomials $H e_{n}(x)$ [4, p. 83], generalised Laguerre polynomials $L_{n}^{(\alpha)}(x)$ [4, p. 84], special generalised Laguerre polynomials $(-1)^{n} L_{n}^{(-\alpha-n)}(x)$. [4, p. 84] and the Gegenbauer or ultraspherical polynomials $C_{n}^{(\alpha)}(x)[4$, p. 83]; there are also hypergeometric polynomials $g_{n}^{(\alpha, \beta)}(x, 1)$ named after Lagrange in [4, p. 85], for which see also [2, (16), p. 247].

Each of these polynomials satisfies a well-known homogeneous linear differential equation of order 2 , for which there will be second, linearly independent, solutions. The problem which we address is that of providing a generating function for a sequence of such second solutions.

We deal in detail with the Hermite polynomials and state corresponding results for the others.

\section{Preliminaries. If}

$$
u(x, t)=\exp \left(x t-\frac{1}{2} t^{2}\right)
$$

then

$$
\frac{\partial u}{\partial x}=t u, \quad \frac{\partial^{2} u}{\partial x^{2}}=t^{2} u, \quad \frac{\partial u}{\partial t}=(x-t) u
$$

and so $w=u(x, t)$ satisfies the equation

$$
\frac{\partial^{2} w}{\partial x^{2}}-x \frac{\partial w}{\partial x}+t \frac{\partial w}{\partial t}=0
$$

Now $u(x, t)$ is an entire function in the variable $t$ so there is an expansion

$$
u(x, t)=\sum_{n=0}^{\infty} u_{n}(x) t^{n}
$$

Received by the editors July 10, 1987. Presented at the mathematical symposium, Dublin Institute for Advanced Studies, April 15, 1987.

1980 Mathematics Subject Classification (1985 Revision). Primary 42C15, 42C10.

Key words and phrases. Generating functions, classical polynomials, orthogonal polynomials. 
where $u_{n}(x)=H e_{n}(x) / n$ !. On applying (2.2) to (2.3), differentiating termwise with respect to $x$ and $t$, we see that for all $t \in \mathbf{C}$

$$
0=\sum_{n=0}^{\infty}\left[u_{n}^{\prime \prime}(x)-x u_{n}^{\prime}(x)+n u_{n}(x)\right] t^{n},
$$

and so for all $n \geq 0, w_{n}(x)=u_{n}(x)$ satisfies

$$
w_{n}^{\prime \prime}(x)-x w_{n}^{\prime}(x)+n w_{n}(x)=0
$$

which is Hermite's differential equation $[1,22.6 .21]$. We seek an expansion

$$
v(x, t)=\sum_{n=0}^{\infty} v_{n}(x) t^{n}
$$

such that, for each fixed $n, u_{n}$ and $v_{n}$ are linearly independent solutions of (2.4).

3. A guiding example. To prompt an approach to this, we consider a similar situation to (2.3) and (2.4) where now

$$
u(x, t)=\frac{1-t \cos x}{1-2 t \cos x+t^{2}}, \quad v(x, t)=\frac{t \sin x}{1-2 t \cos x+t^{2}}
$$

and

$$
u_{n}(x)=\cos n x, \quad v_{n}(x)=\sin n x .
$$

These expansions are easily found from the geometric progression

$$
(1-\varsigma)^{-1}=\sum_{n=0}^{\infty} \varsigma^{n}
$$

on using the polar coordinates $\zeta=t e^{i x}$, where $t$ and $x$ are real, and then using analytic continuation. Then $u_{n}$ and $v_{n}$ are linearly independent solutions of

$$
w_{n}^{\prime \prime}(x)+n^{2} w_{n}(x)=0
$$

which corresponds to (2.4).

Our heuristic approach is to consider whether we know of any other situation in which $u(x, t)$ and $v(x, t)$ in (3.1), or $u_{n}$ and $v_{n}$ in (3.2), or both, are linked. As

$$
\frac{d^{2}}{d t^{2}}(1-t \cos x)=0, \quad \frac{d^{2}}{d t^{2}}(t \sin x)=0
$$

we see from (3.1) that both $u(x, t)$ and $v(x, t)$ satisfy the equation

$$
\frac{d^{2}}{d t^{2}}\left[\left(1-2 t \cos x+t^{2}\right) w\right]=0
$$

which expands to

$$
\left(1-2 t \cos x+t^{2}\right) w^{\prime \prime}(t)+4(-\cos x+t) w^{\prime}(t)+2 w(t)=0 .
$$

If we now apply to this the standard method of seeking for a homogeneous linear differential equation a solution of the form

$$
w(t)=\sum_{n=-\infty}^{\infty} k_{n} t^{n+\sigma}
$$


where $k_{0} \neq 0$ and $k_{n}=0$ for all $n<0$, we find that we need to satisfy the difference equation

$$
\begin{aligned}
(n+\sigma)(n+\sigma-1) k_{n}-2 \cos x & \cdot(n+\sigma)(n+\sigma-1) k_{n-1} \\
& +(n+\sigma)(n+\sigma-1) k_{n-2}=0, \quad n \in \mathbf{Z} .
\end{aligned}
$$

This is trivially satisfied for all $n<0$, and on putting $n=0$ we obtain the indicial equation $\sigma(\sigma-1)=0$, with roots 0 and 1 . As $u(x, 0)=1, u$ is a solution which corresponds to $\sigma=0$ and on inserting this in (3.5) we see that $k_{n}=u_{n}(x)$ in (3.2) satisfies

(3.6) $n(n-1) u_{n}(x)-2 \cos x \cdot n(n-1) u_{n-1}(x)+n(n-1) u_{n-2}(x)=0 \quad(n \geq 1)$, with $u_{-1}(x)=0$. Similarly as $v(x, 0)=0, v$ is a solution for which $\sigma=1$ so on inserting this in (3.5) we see that $k_{n}=v_{n+1}(x)$ in (3.2) satisfies

$$
(n+1) n v_{n+1}(x)-2 \cos x \cdot(n+1) n v_{n}(x)+(n+1) n v_{n-1}(x)=0,
$$

with $v_{-1}(x)=0$, and so

$$
\text { (3.7) } n(n-1) v_{n}(x)-2 \cos x \cdot n(n-1) v_{n-1}(x)+n(n-1) v_{n-2}(x)=0 \quad(n \geq 2) \text {. }
$$

If we inspect (3.6) and (3.7) we see that it is the term $n$ in the coefficient of $u_{n}(x)$ in (3.6) that enables us to accommodate to the stipulation $k_{0} \neq 0$ and so obtain one nontrivial solution of (3.5). Similarly it is the presence of the term $n-1$ in the coefficients of $v_{n}(x)$ and $v_{n-1}(x)$ in (3.7) that enables a second nontrivial solution to be found.

Now returning to our original problem concerning Hermite polynomials, we note that $w=u(x, t)$ in (2.1) satisfies the differential equation

$$
t w^{\prime}(t)+\left(-x t+t^{2}\right) w(t)=0
$$

and for (3.4) to furnish a solution of this we need

$$
(n+\sigma) k_{n}-x k_{n-1}+k_{n-2}=0, \quad n \in \mathbf{Z} \text {. }
$$

If again we seek a solution with $k_{0} \neq 0$ and $k_{n}=0$ for all $n<0$, we get the indicial equation $\sigma=0$ and so

$$
n k_{n}-x k_{n-1}+k_{n-2}=0, \quad n \geq 1
$$

holds for $k_{n}=u_{n}(x)$ in (2.3) for $n \geq 0$, with $u_{-1}(x)=0$. As compared with (3.6) and (3.7) we have $n$ in the coefficient of $k_{n}$ for use when $n=0$ to enable us to get one nontrivial solution, the one which we know, but we have not got a term $n-1$ in the coefficients of $k_{n}$ and $k_{n-1}$ which would allow a second solution. This suggests that we should work with the difference equation

$$
(n+\sigma-1)\left[(n+\sigma) k_{n}-x k_{n-1}+k_{n-2}\right]=0
$$

instead of (3.8).

Now working backwards, we see that the differential equation for which (3.4) will provide a solution if (3.9) holds, is

$$
\left(t \frac{d}{d t}-1\right)\left[t \frac{d w}{d t}+\left(-x t+t^{2}\right) w\right]=0
$$

the solutions to which have the form

$$
w(x, t)=e^{x t-t^{2} / 2}\left[c_{2}+c_{1} \int_{0}^{t} e^{-x s+s^{2} / 2} d s\right]
$$

where $c_{1}$ and $c_{2}$ are arbitrary constants. 
4. Solution for Hermite's equation. With $w(x, t)$ as in (3.10) we now try to show that we again satisfy (2.2) and thus lead on to (2.4). However, instead we are led to a differential equation of order 4 (instead of (2.4)) when $c_{1} \neq 0, c_{2}=0$. For a way out of this disappointment we look at (3.3) and note that although

$$
\frac{d^{2}}{d t^{2}}\left(c_{2}+c_{1} t\right)=0
$$

it is not arbitrary constants that occur there as in each case $c_{1}$ depends on $x$. Accordingly we now take

$$
w(x, t)=e^{x t-t^{2} / 2}\left[c_{2}(x)+c_{1}(x) \int_{0}^{t} e^{-x s+s^{2} / 2} d s\right]
$$

and try to choose $c_{1}(x)$ and $c_{2}(x)$ so that (2.2) is satisfied. If desired the previous work can be regarded as motivation, and the calculations carefully checked only from here on.

From (4.1)

$$
\frac{\partial w}{\partial x}=t w+e^{x t-t^{2} / 2}\left[c_{2}^{\prime}(x)+c_{1}^{\prime}(x) \int_{0}^{t} e^{-x s+s^{2} / 2} d s-c_{1}(x) \int_{0}^{t} s e^{-x s+s^{2} / 2} d s\right]
$$

and on integration by parts

$$
\begin{aligned}
\frac{\partial w}{\partial x}=t w+e^{x t-t^{2} / 2}\left\{c_{2}^{\prime}(x)\right. & +c_{1}(x)-c_{1}(x) e^{-x t+t^{2} / 2} \\
& \left.+\left[c_{1}^{\prime}(x)-x c_{1}(x)\right] \int_{0}^{t} e^{-x s+s^{2} / 2} d s\right\}
\end{aligned}
$$

We choose $c_{1}(x)$ to satisfy

$$
c_{1}^{\prime}(x)-x c_{1}(x)=0
$$

so that we can take

$$
c_{1}(x)=e^{x^{2} / 2}
$$

We next choose $c_{2}(x)$ so that

$$
c_{2}^{\prime}(x)+c_{1}(x)=0
$$

and so can take

$$
c_{2}(x)=-\int_{0}^{x} e^{s^{2} / 2} d s .
$$

With this choice in (4.1) we get in (4.2) that

$$
\frac{\partial w}{\partial x}=t w-c_{1}(x)=t w-e^{x^{2} / 2} .
$$

From this

so that

$$
\frac{\partial^{2} w}{\partial x^{2}}=t \frac{\partial w}{\partial x}-x e^{x^{2} / 2}=t^{2} w-(t+x) e^{x^{2} / 2}
$$

But by (4.1),

$$
\frac{\partial^{2} w}{\partial x^{2}}-x \frac{\partial w}{\partial x}=t^{2} w-x t w-t e^{x^{2} / 2}
$$

$$
\frac{\partial w}{\partial t}=(x-t) w+c_{1}(x)
$$


so that

and we have (2.2) again. Accordingly in

$$
t \frac{\partial w}{\partial t}=x t w-t^{2} w+t e^{x^{2} / 2}=-\frac{\partial^{2} w}{\partial x^{2}}+x \frac{\partial w}{\partial x}
$$

$$
\begin{aligned}
v(x, t) & =e^{x t-t^{2} / 2}\left[-\int_{0}^{x} e^{s^{2} / 2} d s+e^{x^{2} / 2} \int_{0}^{t} e^{-x s+s^{2} / 2} d s\right] \\
& =\sum_{n=0}^{\infty} v_{n}(x) t^{n}
\end{aligned}
$$

the coefficients $v_{n}$ satisfy the Hermite differential equation (2.4).

5. Linear independence. A pair of linearly independent solutions of the equation (2.4) is $M\left(-\frac{1}{2} n, \frac{1}{2} ; \frac{1}{2} x^{2}\right), x M\left(\frac{1}{2}-\frac{1}{2} n, \frac{3}{2} ; \frac{1}{2} x^{2}\right)$ where

$$
M(a, b ; z)=\sum_{m=0}^{\infty} \frac{(a)_{m}}{m !(b)_{m}} z^{m}
$$

is the well-known Kummer function $[1,13.1 .2]$. Accordingly any solution $W(x)$ of (2.4), in a neighbourhood of the origin, can be expressed as a linear combination of these,

$$
W(x)=c_{1} M\left(-\frac{1}{2} n, \frac{1}{2} ; \frac{1}{2} x^{2}\right)+c_{2} x M\left(\frac{1}{2}-\frac{1}{2} n, \frac{3}{2} ; \frac{1}{2} x^{2}\right)
$$

for some constants $c_{1}$ and $c_{2}$, and in fact

$$
c_{1}=W(0), \quad c_{2}=W^{\prime}(0) .
$$

This can be applied to $u(x, t)$ in (2.1) to prove the results $[1,22.5 .56-57]$ that for $m \geq 0$

$$
\begin{aligned}
u_{2 m}(x) & =\frac{(-1 / 2)^{m}}{m !} M\left(-m, \frac{1}{2} ; \frac{1}{2} x^{2}\right) \\
u_{2 m+1}(x) & =\frac{(-1 / 2)^{m}}{m !} x M\left(-m, \frac{1}{2} ; \frac{1}{2} x^{2}\right) .
\end{aligned}
$$

Now from (4.4)

$$
\begin{aligned}
v(0, t) & =e^{-t^{2} / 2} \int_{0}^{t} e^{s^{2} / 2} d s=t e^{-t^{2} / 2} \int_{0}^{1} e^{t^{2} r^{2} / 2} d r \\
& =t \sum_{m=0}^{\infty} \frac{\left(-t^{2} / 2\right)^{m}}{m !}\left[\int_{0}^{1}\left(1-r^{2}\right)^{m} d r\right]
\end{aligned}
$$

The integral in this is equal to

$$
\frac{1}{2} \int_{0}^{1} y^{-1 / 2}(1-y)^{m} d y=\frac{1}{2} B\left(\frac{1}{2}, m+1\right)=\frac{m !}{(3 / 2)_{m}}
$$

Hence

$$
v(0, t)=\sum_{m=0}^{\infty} \frac{(-1)^{m}}{2^{m}(3 / 2)_{m}} t^{2 m+1}
$$

so that

$$
v_{n}(0)= \begin{cases}\frac{(-1 / 2)^{m}}{(3 / 2)_{m}}, & \text { if } n=2 m+1 \\ 0, & \text { if } n=2 m\end{cases}
$$


Moreover from (4.3) and (4.4)

$$
\begin{aligned}
\left.\frac{\partial}{\partial x} v(x, t)\right|_{x=0} & =\sum_{n=0}^{\infty} v_{n}^{\prime}(0) t^{n}=t v(0, t)-1 \\
& =-1+\sum_{m=0}^{\infty} \frac{(-1)^{m}}{2^{m}(3 / 2)_{m}} t^{2 m+2}
\end{aligned}
$$

so that

$$
v_{n}^{\prime}(0)= \begin{cases}0, & \text { if } n=2 m+1 \\ -1, & \text { if } n=0 \\ \frac{(-1)^{m}}{2^{m}(3 / 2)_{m}}, & \text { if } n=2 m+2\end{cases}
$$

From (5.1), (5.3) and (5.4) it follows that

$$
\begin{aligned}
& v_{2 m+2}(x)=\frac{(-1)^{m}}{2^{m}(3 / 2)_{m}} x M\left(-m-\frac{1}{2}, \frac{3}{2} ; \frac{1}{2} x^{2}\right) \\
& v_{2 m+1}(x)=\frac{(-1)^{m}}{2^{m}(3 / 2)_{m}} M\left(-m-\frac{1}{2}, \frac{1}{2} ; \frac{1}{2} x^{2}\right) .
\end{aligned}
$$

These give $v_{n}(x)$ explicitly, and by comparison with (5.2) show that $u_{n}$ and $v_{n}$ are linearly independent.

6. Statement of analogous results. By a similar method we can find and establish the following.

With the same notation, for

$$
u(x, t)=\exp \left[-\frac{x t}{1-t}\right](1-t)^{-\alpha-1}
$$

we have

$$
u_{n}(x)=L_{n}^{(\alpha)}(x),
$$

the generalised Laguerre polynomials. Then $u(x, t)$ satisfies the equation

$$
x \frac{\partial^{2} w}{\partial x^{2}}+(1+\alpha-x) \frac{\partial w}{\partial x}+t \frac{\partial w}{\partial t}=0
$$

and $u_{n}(x)$ satisfies

$$
x w_{n}^{\prime \prime}(x)+(1+\alpha-x) w_{n}^{\prime}(x)+n w_{n}(x)=0 ;
$$

see $[1,22.6 .15]$. Then

$$
\begin{aligned}
v(x, t)=(1-t)^{-\alpha-1} \exp \left[-\frac{x t}{1-t}\right]\{ & \int_{0}^{x} e^{s} s^{-\alpha-1} d s \\
& \left.+x^{-\alpha} e^{x} \int_{0}^{t}(1-s)^{\alpha-1} \exp \left[\frac{x s}{1-s}\right] d s\right\}
\end{aligned}
$$

also satisfies (6.1) and the corresponding $v_{n}(x)$ satisfy (6.2).

For

$$
u(x, t)=(1-t)^{-\alpha} e^{x t}
$$


we have

Then $u(x, t)$ satisfies

$$
u_{n}(x)=(-1)^{n} L_{n}^{(-\alpha-n)}(x)
$$

$$
x \frac{\partial^{2} w}{\partial x^{2}}+(1-\alpha-x) \frac{\partial w}{\partial x}+t \frac{\partial w}{\partial t}-t \frac{\partial^{2} w}{\partial t \partial x}=0
$$

and $u_{n}(x)$ satisfies

$$
x w_{n}^{\prime \prime}(x)+(1-\alpha-n-x) w_{n}^{\prime}(x)+n w_{n}(x)=0 .
$$

To this we can add that

$$
v(x, t)=(1-t)^{-\alpha} e^{x t}\left[-x^{-\alpha} e^{-x} \int_{0}^{x} s^{\alpha-1} e^{s} d s+\int_{0}^{t}(1-s)^{\alpha-1} e^{-x s} d s\right]
$$

also satisfies (6.3) and the corresponding $v_{n}(x)$ satisfy (6.4).

For

$$
u(x, t)=(1-x t)^{-\alpha}(1-t)^{-\beta}
$$

we have (cf. [4, p. 452, Problem 25])

$$
u_{n}(x)=g_{n}^{(\alpha, \beta)}(x, 1)=\frac{(\beta)_{m}}{m !} F(-n, \alpha ; 1-\beta-n ; x),
$$

where $F(a, b ; c ; z)$ is the hypergeometric function $[1$, p. 556]. Then $u(x, t)$ satisfies

$$
x(1-x) \frac{\partial^{2} w}{\partial x^{2}}+[1-\beta-(1+\alpha) x] \frac{\partial w}{\partial x}+\alpha t \frac{\partial w}{\partial t}-(1-x) t \frac{\partial^{2} w}{\partial t \partial x}=0
$$

and $u_{m}(x)$ satisfies

$$
x(1-x) u_{n}^{\prime \prime}(x)+[1-\beta-n-(1+\alpha-n) x] u_{n}^{\prime}(x)+\alpha n u_{n}(x)=0 .
$$

To this we can add that

$$
\begin{aligned}
v(x, t)=(1-x t)^{-\alpha}(1-t)^{-\beta}[ & -\int_{0}^{x} s^{\beta-1}(1-s)^{-\alpha-\beta} d s \\
& \left.+x^{\beta}(1-x)^{1-\alpha-\beta} \int_{0}^{t}(1-x s)^{\alpha-1}(1-s)^{\beta-1} d s\right]
\end{aligned}
$$

also satisfies (6.5) and the corresponding $v_{n}(x)$ satisfy (6.6).

Finally for

we have

$$
u(x, t)=\left(1-2 x t+t^{2}\right)^{-\alpha}
$$

$$
u_{n}(x)=C_{n}^{(\alpha)}(x)
$$

the Gegenbauer or ultraspherical polynomials. Then $u(x, t)$ satisfies

$$
\left(1-x^{2}\right) \frac{\partial^{2} w}{\partial x^{2}}-(2 \alpha+1) x \frac{\partial w}{\partial x}+(2 \alpha+1) t \frac{\partial w}{\partial t}+t^{2} \frac{\partial^{2} w}{\partial t^{2}}=0
$$

and $u_{n}(x)$ satisfies

$$
\left(1-x^{2}\right) w_{n}^{\prime \prime}(x)-(2 \alpha+1) x w_{n}^{\prime}(x)+n(2 \alpha+n) w_{n}(x)=0
$$

see $[1,22.6 .5]$. To this we can add that

$$
\begin{aligned}
v(x, t)=\left(1-2 x t+t^{2}\right)^{-\alpha}\{ & -\int_{0}^{x}\left(1-s^{2}\right)^{-\alpha-1 / 2} d s \\
& \left.+\left(1-x^{2}\right)^{-\alpha+1 / 2} \int_{0}^{t}\left(1-2 x s+s^{2}\right)^{\alpha-1} d s\right\}
\end{aligned}
$$

also satisfies (6.7) and the corresponding $v_{n}(x)$ satisfy (6.8). 
7. Concluding remark. The technique is somewhat general, as we can try to apply it whenever $u(x, t)$ satisfies a first order homogeneous linear differential equation in $t$, with polynomial coefficients.

\section{REFERENCES}

1. M. Abramowitz and I. A. Stegun, Handbook of mathematical functions, Dover, New York, 1965.

2. A. Erdélyi, W. Magnus, F. Oberhettinger and F. G. Tricomi, Higher transcendental functions, vol. III, McGraw-Hill, New York, 1955.

3. E. B. McBride, Obtaining generating functions, Springer-Verlag, Berlin and New York, 1971.

4. H. M. Srivastava and H. L. Manocha, A treatise on generating functions, Ellis Horwood and Wiley, New York, 1984.

Department of Mathematics, University College, Cork, IREland 